\title{
Carnosine suppresses oxygen-glucose deprivation/ recovery-induced proliferation and migration of reactive astrocytes of rats in vitro
}

\author{
Li OU-YANG ${ }^{1}$, Yuan LIU $^{1}$, Bing-yu WANG ${ }^{1}$, Pei CAO ${ }^{1}$, Jing-jing ZHANG ${ }^{1}$, Yu-yan HUANG ${ }^{1}$, Yao SHEN ${ }^{1, *}$, Jian-xin LYU $^{1,2}$ \\ ${ }^{1}$ Key Laboratory of Laboratory Medicine, Ministry of Education, School of Laboratory Medicine and Life sciences, Wenzhou Medical \\ University, Wenzhou 325035, China; ${ }^{2}$ Laboratory Medicine College, Hangzhou Medical College, Hangzhou 310053, China
}

\begin{abstract}
Glial scar formation resulted from excessive astrogliosis limits axonal regeneration and impairs recovery of function, thus an intervention to ameliorate excessive astrogliosis is crucial for the recovery of neurological function after cerebral ischemia. In this study we investigated the effects of carnosine, an endogenous water-soluble dipeptide ( $\beta$-alanyl-L-histidine), on astrogliosis of cells exposed to oxygen-glucose deprivation/recovery $(\mathrm{OGD} / \mathrm{R})$ in vitro. Primary cultured rat astrocytes exhibited a significant increase in proliferation at $24 \mathrm{~h}$ recovery after OGD for $2 \mathrm{~h}$. Pretreatment with carnosine ( $5 \mathrm{mmol} / \mathrm{L})$ caused $\mathrm{G}_{1}$ arrest of reactive astrocytes, significantly attenuated OGD/R-induced increase in cyclin D1 protein expression and suppressed OGD/R-induced proliferation of reactive astrocytes. Carnosine treatment also reversed glycolysis and ATP production, which was elevated at $24 \mathrm{~h}$ recovery after OGD. A marked increase in migration of reactive astrocytes was observed at $24 \mathrm{~h}$ after OGD, whereas carnosine treatment reversed the expression levels of MMP-9 and suppressed the migration of astrocytes. Furthermore, carnosine also improved neurite growth of cortical neurons co-cultured with astrocytes under ischemic conditions. These results demonstrate that carnosine may be a promising candidate for inhibiting astrogliosis and promoting neurological function recovery after ischemic stroke.
\end{abstract}

Keywords: cerebral ischemia; astrogliosis; carnosine; oxygen-glucose deprivation/recovery (OGD/R); cell cycle; energy metabolism; neurite growth

Acta Pharmacologica Sinica (2018) 39: 24-34; doi: 10.1038/aps.2017.126; published online 21 Sep 2017

\section{Introduction}

Cerebral ischemic stroke is a highly disabling and deadly disease worldwide ${ }^{[1]}$. Although a portion of patients with stroke can exhibit long-term survival, they will have lifelong disability owing to the low axonal regenerative capacity of the adult central nervous system (CNS) ${ }^{[2]}$. The lack of axonal regeneration during the chronic phase following cerebral ischemia is due not to the intrinsic incapacity of the neuron to regenerate but rather to the presence of an adverse environment in the injury ${ }^{[3]}$.

Glial scars predominately consist of reactive astrocytes, microglia/macrophages and extracellular matrix molecules that are known to be secreted by reactive astrocytes ${ }^{[4,5]}$. Glial scar formation shows polarity towards the injury ${ }^{[6]}$ and is beneficial in limiting the expansion of injury. However, it

\footnotetext{
* To whom correspondence should be addressed.

E-mail yueshen-2002@163.com

Received 2017-02-24 Accepted 2017-06-05
}

ultimately limits axonal regeneration and impairs recovery of function $^{[7,8]}$. In this regard, manipulation of the glial scar may be favorable for neuronal regeneration and functional recovery.

$L$-Carnosine ( $\beta$-alanyl- $L$-histidine) is an endogenous watersoluble dipeptide that can be transported from the plasma to the cerebrospinal fluid through a proton-coupled oligopeptide transporter known as PEPT2 ${ }^{[9,10]}$. In the CNS of vertebrates, carnosine is mainly distributed in glial cells, especially in astrocytes $^{[11]}$. Carnosine is a very versatile dipeptide, and it has been postulated to have numerous biological roles such as free radical scavenger, protein glycosylation inhibitor, antiinflammatory agent and $\mathrm{pH}$ buffer ${ }^{[12]}$. However, so far, the physiological activity of carnosine in the brain remains to be elucidated. Recently, data obtained in several independent laboratories suggested that carnosine can provide neuroprotection against ischemic injury in the acute phase of stroke $\mathrm{e}^{[13-15]}$. However, the role of carnosine in glial scar formation following cerebral ischemia remains unclear. Recent studies from our group and other laboratories have revealed that by inhib- 
iting bioenergy production, carnosine inhibits the proliferation of several cancer cell lines ${ }^{[16,17]}$. In addition, we found that carnosine has dual effects on the energy metabolism of cultured astrocytes under physiological and ischemic conditions ${ }^{[18,19]}$. Thus, we note that carnosine may have an influence on glial scar formation in response to cerebral ischemia by affecting cell proliferation.

Therefore, the current study was designed to explore whether carnosine could inhibit gliosis and promote neuronal regeneration after ischemia in an in vitro ischemic model and the possible underlying mechanisms.

\section{Materials and methods Materials}

Carnosine ( $\beta$-alanyl- $L$-histidine), bovine serum albumin, Triton X-100, 5-bromodeoxyuridine, propidium iodide, RNase, sodium pyruvate, rotenone, oligomycin, and carbonyl cyanide p-trifluoromethoxyphenylhydrazone (FCCP) were obtained from Sigma (St Louis, MO, USA). Dulbecco's modified Eagle's medium (DMEM), glucose-free DMEM, fetal bovine serum, horse serum, and B27 were from GIBCO-BRL (Grand Island, NY, USA). Trypsin, poly-D-lysine, penicillin, streptomycin, L-glutamine, lactate dehydrogenase kit, paraformaldehyde, and BCA Protein Assay Kit were purchased from Beyotime Institute of Biotechnology (Nanjing, China). XF assay medium and XF calibrant solution were purchased from Seahorse Bioscience. A rat interleukin $1 \beta$ (IL-1 $\beta$ ) ELISA kit was purchased from Nanjing Jiancheng Bioengineering Institute (China).

\section{Primary cortical astrocyte-enriched culture}

All experiments using animals were performed in accordance with the National Institutes of Health Guide for the Care and Use of Laboratory Animals. Primary cultures of cortical astrocytes were prepared from the cortices of neonatal SpragueDawley rats as described previously ${ }^{[18]}$. In brief, cortices were dissected from the brains under sterile conditions, digested in $0.25 \%$ trypsin for $20 \mathrm{~min}$ at $37^{\circ} \mathrm{C}$, and then the dissociated cells were seeded in high glucose $(4.5 \mathrm{~g} / \mathrm{L})$ DMEM supplemented with $10 \%$ fetal bovine serum, $2 \mathrm{mmol} / \mathrm{L}$ glutamine, $100 \mathrm{U} / \mathrm{mL}$ penicillin and $100 \mu \mathrm{g} / \mathrm{mL}$ streptomycin. The cultures were maintained at $37^{\circ} \mathrm{C}$ in a humidified atmosphere of $5 \% \mathrm{CO}_{2} / 95 \%$ air. On d 10-11, the confluent cultures were shaken overnight to minimize microglia contamination. More than $95 \%$ of the cultured cells were astrocytes as identified by immunofluorescent staining for GFAP.

\section{Neuron/astrocyte co-cultures}

Neuron/astrocyte co-cultures were prepared from 1-d-old Sprague-Dawley rat cortices. In brief, the cerebral cortices were digested with $0.125 \%$ trypsin for $10 \mathrm{~min}$ at $37^{\circ} \mathrm{C}$, and the dissociated cells were seeded at a density of $0.5 \times 10^{5}$ cells $/ \mathrm{cm}^{2}$ in 96-well plates or $25 \mathrm{~cm}^{2}$ flasks previously coated with poly$D$-lysine. Cells were cultured in high glucose $(4.5 \mathrm{~g} / \mathrm{L})$ DMEM supplemented with $7.5 \%$ fetal bovine serum, $7.5 \%$ horse serum, $2 \mathrm{mmol} / \mathrm{L}$ glutamine, 100 units/mL penicillin and 100 $\mu \mathrm{g} / \mathrm{mL}$ streptomycin and were maintained in a humidified
$\mathrm{CO}_{2}$ incubator $\left(5 \% \mathrm{CO}_{2}, 95 \%\right.$ air, $\left.37^{\circ} \mathrm{C}\right)$. After $48 \mathrm{~h}$ in vitro, the culture medium was half replaced with special culture medium supplemented with B-27. The medium was changed every 2-3 d. After 10-12 d, the neurons in these cultures sat on the top of a confluent monolayer of astrocytes. The experiments were performed using these cultures.

\section{Oxygen-glucose deprivation and carnosine treatment}

The cells were washed twice and incubated in glucose-free DMEM. Then, the cells were transferred into an anaerobic chamber filled with a gas mixture of $95 \% \mathrm{~N}_{2}$ and $5 \% \mathrm{CO}_{2}$ at $37^{\circ} \mathrm{C}$. At the end of OGD, the cells were replaced with normal culture medium and returned to the normal culture condition for an additional 24,48 or $72 \mathrm{~h}$. In each experiment, cultures exposed to OGD were compared with normoxic controls supplied with DMEM containing glucose and maintained in standard incubation conditions. Carnosine at a concentration of 1 or $5 \mathrm{mmol} / \mathrm{L}$ was supplied $30 \mathrm{~min}$ before OGD and was present throughout the OGD and recovery process.

\section{Lactate dehydrogenase (LDH) assay protocol}

Relative cell proliferation was determined by comparing the total amount of LDH present in cell lysates after 2- or 4-h OGD followed by $24-, 48$ - or 72-h recovery to LDH present in cell lysates of controls as described previously ${ }^{[20]}$. Total LDH present in cell lysates was measured with an LDH kit according to the manufacturer's instructions. LDH metabolism was quantified by measuring absorbance at $490 \mathrm{~nm}$ and $600 \mathrm{~nm}$ in a Thermo Scientific Varioskan Flash.

\section{BrdU immunofluorescent staining}

To further assess proliferation of glial cells in culture, cells were incubated with $30 \mu \mathrm{mol} / \mathrm{L}$ 5-bromodeoxyuridine (BrdU) after OGD. After the experiments, the cells were fixed with $4 \%$ paraformaldehyde for $10 \mathrm{~min}$ and then were washed in PBS for $3 \mathrm{~min}$, and the DNA was denatured by incubating in $2 \mathrm{~mol} / \mathrm{L} \mathrm{HCl}$ for $0.5 \mathrm{~h}$. After being washed twice in $0.1 \mathrm{~mol} / \mathrm{L}$ borate buffer ( $\mathrm{pH} 8.5$ ) and three times in PBS, cells were blocked with $3 \%$ BSA for $1 \mathrm{~h}$. Then, cells were incubated in mouse monoclonal antibody against $\operatorname{BrdU}(1: 600, \mathrm{CST}, \mathrm{USA})$ overnight. After being washed twice with PBS, cells were then incubated with goat anti-mouse IgG (1:300, Invitrogen, USA) for $0.5 \mathrm{~h}$. After further washing in PBS, cells were mounted and observed under fluorescence microscopy (Nikon Eclipse TI, Japan). Cell number was counted by NIH ImageJ software.

\section{Immunocytochemistry}

Immunostaining was also performed in cultured astrocytes and neurons. Cells seeded on coverslips were fixed with $4 \%$ paraformaldehyde for $10 \mathrm{~min}$ and incubated in 3\% bovine serum albumin containing $0.1 \%$ Triton $\mathrm{X}-100$ for $1 \mathrm{~h}$. Then, primary antibodies in 3\% BSA were applied for $1 \mathrm{~h}$ or overnight. For astrocytes and neurons, mouse anti-GFAP (1:300, CST, USA), rabbit anti-Neu (1:200, Abcam, USA), and rabbit anti-beta III Tubulin (1:200, Abcam, USA) were used. After repeated washes in PBS, cells were incubated with Cy3- 
labeled goat anti-mouse IgG (1:300, Invitrogen, USA) or Alexa Fluor 488-labeled goat anti-rabbit IgG (1:300, Invitrogen, USA) for $0.5 \mathrm{~h}$ at $37^{\circ} \mathrm{C}$. After further washing in PBS, cultures were dried, coverslipped, and mounted on glass slides. Finally, the stained cells were observed under fluorescence microscopy (Nikon Eclipse TI, Japan).

\section{Enzyme-linked immunosorbent assay (ELISA) for IL-1 $\beta$}

After OGD/R and carnosine treatment, the supernatants of the cultures were collected and stored at $-80{ }^{\circ} \mathrm{C}$ until the measurement of IL-1 $\beta$ concentrations. This was done according to the manufacturer's protocol using commercial rat ELISA kits for IL-1 $\beta$. Absorbance was measured on a microplate reader (Thermo Scientific Varioskan Flash). IL-1 $\beta$ level was determined using standard curves method.

\section{Determination of ATP production}

The ATP assay was performed as described previously ${ }^{[13]}$. Briefly, the cells were lysed with a lysis buffer followed by centrifugation at $10000 \times g$ for $2 \mathrm{~min}$ at $4{ }^{\circ} \mathrm{C}$. Finally, in 6-well plates, the level of ATP was determined by mixing $20 \mu \mathrm{L}$ of the supernatant with $100 \mu \mathrm{L}$ of luciferase reagent, which catalyzed the light production from ATP and luciferin. Luminance was measured by a monochromator microplate reader. Standard curves were also generated and the protein concentration of each group was determined using the BCA protein assay kit. Total ATP levels were expressed as $\mathrm{nmol} / \mathrm{mg}$ protein.

\section{Cell migration assay}

Cell migration was assayed using fibronectin-coated polycarbonate filters $(8-\mu \mathrm{m}$ pore size, Transwell; Becton Dickinson, USA). In brief, $1 \times 10^{5}$ cells/wells containing $1 \%$ FBS medium were added to the top compartment. The lower chambers were filled with $600 \mu \mathrm{L}$ of DMEM supplemented with $10 \%$ FBS. The astrocytes were allowed to migrate for $48 \mathrm{~h}$; then, the filters were washed with PBS, fixed with ice-cold $4 \%$ paraformaldehyde and stained with DAPI, and observed under fluorescence microscopy (Nikon Eclipse TI, Japan).

\section{Western blot analysis}

Western blot analysis was carried out by standard protocol. The following antibodies were used: mouse anti-GAPDH (Abcam, 1:1000), mouse anti-tublin (Abcam, 1:1000), mouse anti-GFAP (CST, 1:1000), rabbit anti-cyclin D1 (Abcam, 1:1000), rabbit anti-MMP 9 (Abcam, 1:1000), and horseradish-conjugated goat anti-mouse antibody or horseradish-conjugated goat anti-rabbit antibody.

\section{Cell cycle analysis}

Cell cycle analysis was performed by propidium iodide (PI) staining. Astrocytes were trypsinized and washed with icecold PBS (pH 7.4) and fixed in ice-cold 70\% ethanol. The cells were then washed with PBS, treated with $500 \mathrm{U} / \mathrm{mL}$ RNase at $37^{\circ} \mathrm{C}$ for $30 \mathrm{~min}$, and finally stained with PI in PBS. Ten thousand cells were counted for each data point. Cell cycle analysis was performed using a flow cytometer.

\section{Extracellular flux technology}

The oxygen consumption rates (OCRs) of astrocytes in different conditions were measured using a Seahorse XF96 Extracellular Flux Analyzer. This instrument allows for the sensitive measurement of glycolysis and multiple parameters of mitochondrial function, including basal OCR, spare respiratory capacity, maximal OCR, ATP-linked respiration and proton leak from adherent intact cultured cells. After baseline measurements, OCRs were measured after sequentially adding to each well oligomycin $(1 \mu \mathrm{g} / \mathrm{mL})$, FCCP $(1 \mu \mathrm{mol} / \mathrm{L})$ and rotenone $(1 \mu \mathrm{mol} / \mathrm{L})$. All assays were conducted using a seeding density of $1 \times 10^{4}$ cells/well in $200 \mathrm{~L}$ of DMEM in a poly-D-lysine-coated XF 96-cell culture microplate. The cells were switched to unbuffered DMEM (carnosine was absent) supplemented with $2 \mathrm{mmol} / \mathrm{L}$ sodium pyruvate $1 \mathrm{~h}$ prior to the beginning of the assay and maintained at $37^{\circ} \mathrm{C}$.

\section{Neurite growth quantification}

The lengths of neurites in each neuron were traced manually and quantified using NIH ImageJ software. Mean neurite length for each neuron was determined for 50-100 neurons per condition from three separate experiments.

\section{Statistical analysis}

Statistical analyses were performed using SPSS 11.5 for Windows. All data were presented as the mean \pm SD. One-way ANOVA (analysis of variance) followed by LSD (least significant difference) or Dunnett's T3 post hoc test (where equal variances were not assumed) was applied for multiple comparisons, whereas Student's $t$-test was used for comparisons between two groups. $P<0.05$ was taken to indicate statistically significant effects.

\section{Results}

Effect of OGD/R times on the proliferation of cultured astrocytes

Total LDH content assay and BrdU labeling were used to explore appropriate OGD/R time points that can induce astrocytes to present glial scar characteristics. Combined with the previous experiments by our group ${ }^{[18,21]}$, two intermediate durations of OGD and three recovery time points were tested. We found that the total LDH content of astrocytes returned to the control level when exposed to 4-h OGD followed by 24-h recovery, while 2-h OGD and 24-h recovery induced a significant increase in total LDH content (Figure 1A). Next, we tested three recovery time points with 2-h OGD treatment. The total amount of LDH present in cell lysates was $139 \%$ of controls after $24-\mathrm{h}$ recovery, but with recovery for 48 $\mathrm{h}$ and $72 \mathrm{~h}$, the total amounts of LDH were not significantly different from the controls (Figure 1B). Simultaneously, for further verification, BrdU immunostaining was also carried out. The results showed that OGD for $2 \mathrm{~h}$ and recovery for 24 $h$ could induce the most obvious astrogliosis (Figure 1C, 1D), which was consistent with the LDH content detection results. To further ensure that OGD/R caused reactive astrocytes, immunohistochemistry and Western blot analysis were also performed to confirm the high expression of GFAP, which has 
A

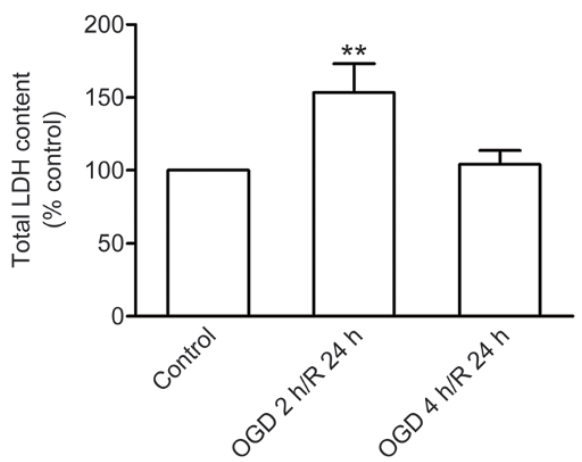

C

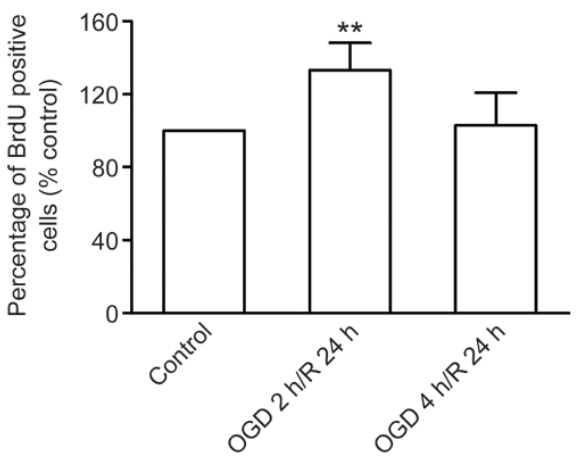

E
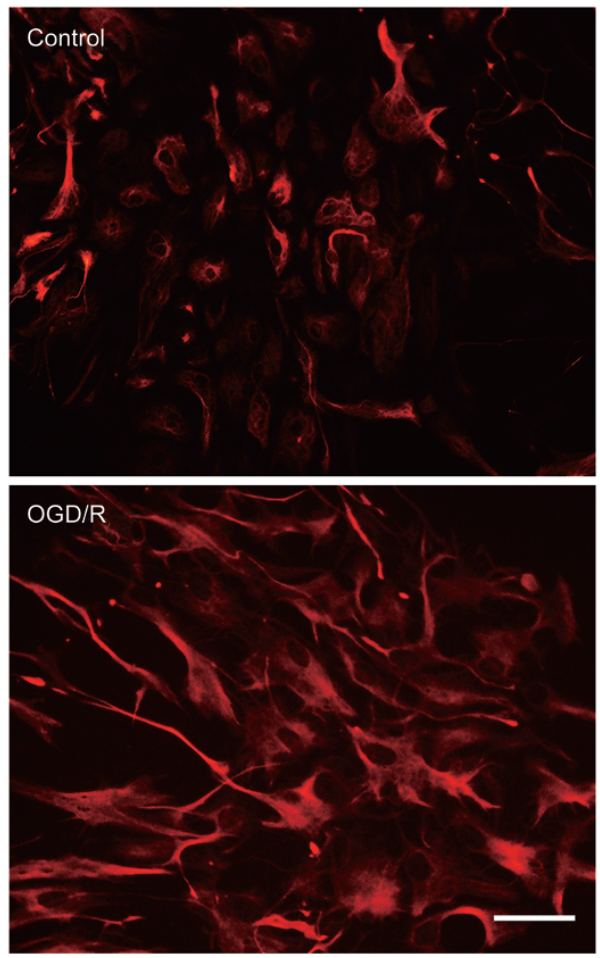

B

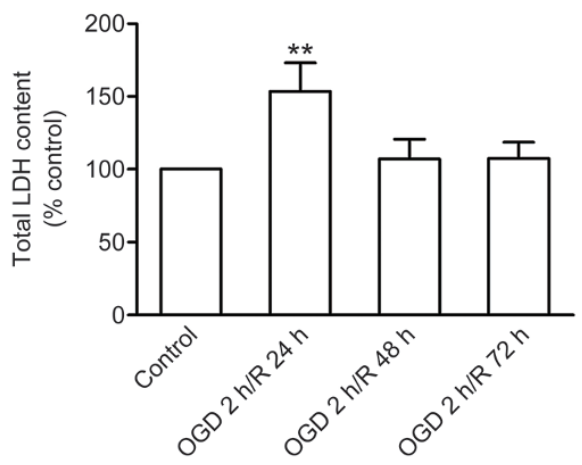

D

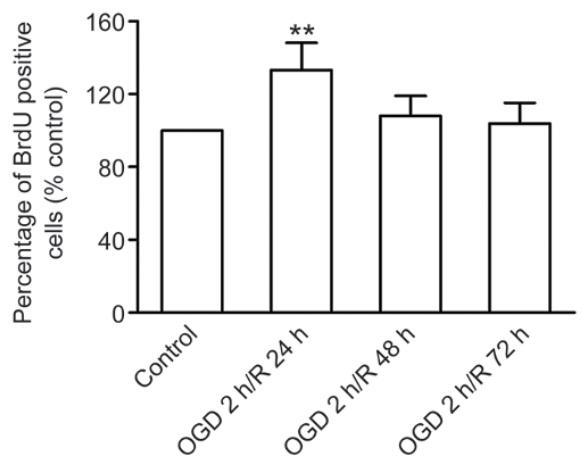

F

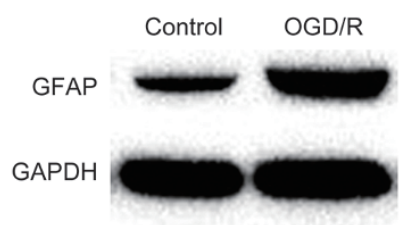

G

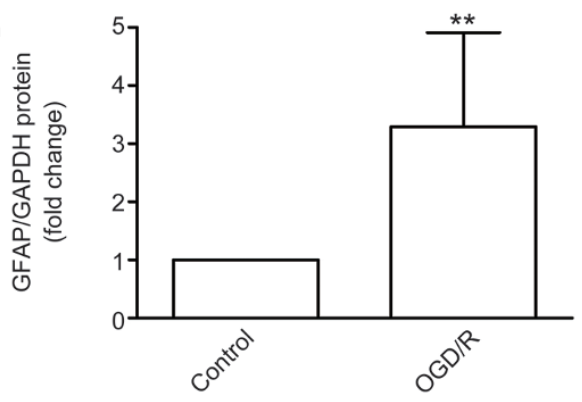

Figure 1. Effect of OGD and recovery times on the proliferation of cultured astrocytes. Astrocytes were exposed to OGD for 2 or $4 \mathrm{~h}$ followed by recovery for 24,48 or $72 \mathrm{~h}$. Total LDH content was assayed after OGD for 2 or $4 \mathrm{~h}$ followed by recovery for $24 \mathrm{~h}$ (A) or after OGD for $2 \mathrm{~h}$ followed by recovery for 24,48 or $72 \mathrm{~h}$ (B). The percentage of BrdU-positive cells was statistically analyzed after OGD for 2 or $4 \mathrm{~h}$ followed by recovery for $24 \mathrm{~h}$ (C) or after OGD for $2 \mathrm{~h}$ followed by recovery for 24,48 or $72 \mathrm{~h}(\mathrm{D})$. Immunohistochemistry for GFAP expression in cultured astrocytes exposed to OGD for $2 \mathrm{~h}$ and recovery for $24 \mathrm{~h}(\mathrm{E})$. ( $\mathrm{F}, \mathrm{G})$ Western blot of GFAP expression in cultured astrocytes exposed to OGD for $2 \mathrm{~h}$ and recovery for $24 \mathrm{~h}$. Data represent the mean \pm SD. ${ }^{* *} P<0.01$ vs control group. Scale bar, $100 \mu \mathrm{m}$.

been recognized as a characteristic of reactive astrocytes. We found that the expression of GFAP was increased remarkably after 2-h OGD and 24-h recovery (Figure 1E and 2D, 2E). In addition, we found that the released IL- $1 \beta$, one of the most 
extensively studied proinflammatory cytokines that has been suggested to play an important role in astrogliosis ${ }^{[22]}$, was markedly increased (169\% of control, Figure $2 \mathrm{~F}$ ) after OGD/R treatment. Thus, the duration of $2 \mathrm{~h}$ for OGD and $24 \mathrm{~h}$ for recovery was used in the following experiments.

\section{Carnosine suppressed reactive gliosis of astrocytes induced by} $\mathrm{OGD} / \mathrm{R}$ in vitro

The effect of carnosine on the reactive gliosis of cultured astrocytes was determined via total LDH content detection and BrdU staining. Compared with the OGD/R group, treatment with 1 and $5 \mathrm{mmol} / \mathrm{L}$ carnosine significantly decreased the total amount of LDH present in cell lysates (Figure 2A). In the BrdU staining assay, we found that the percentage of BrdU-positive astrocytes was significantly decreased in the carnosine-treated group (OGD/R group: $133 \% \pm 14.9 \%$; Carnosine+OGD/R group: $110 \% \pm 15.7 \%, P<0.01$; Figure $2 B$, $2 \mathrm{C})$. The change of the expression level of GFAP protein in the astrocytes under the influence of $5 \mathrm{mmol} / \mathrm{L}$ carnosine was also determined by Western blotting, and the results showed that carnosine treatment partly reversed the increase in GFAP expression induced by OGD/R (Figure 2D, 2E). In addition, the OGD/R-induced increase in IL- $1 \beta$ release by astrocytes was reversed by carnosine treatment (OGD/R group: 1.77 \pm 0.25 ; Carnosine+OGD/R group: $1.23 \pm 0.21, P<0.05$; Figure $2 \mathrm{~F}$ ), indicating that the functional properties of reactive astrocytes were altered by carnosine. Thus, all the data suggested that carnosine may suppress the reactive gliosis of astrocytes induced by OGD/R.

Carnosine inhibited cell cycle progression and regulated the expression of cyclin D1 proteins in astrocytes exposed to OGD/R

To explore whether the inhibitory effect of carnosine on the proliferation of astrocytes is related to its action on the cell cycle, we performed a flow cytometry assay (FACS). As shown in Figure 3A and 3B, OGD/R significantly decreased the number of cells in $G_{1}$ phase and increased the number of cells in $S$ phase. Carnosine treatment almost completely reversed the decrease in the number of cells in $G_{1}$ phase (OGD/R group: $74.1 \% \pm 1.82 \%$, Carnosine+OGD/R group: $81.2 \% \pm 2.07 \%, P<0.05)$ and the increase in the number of cells in $S$ phase (OGD/R group: $16.8 \% \pm 1.98 \%$, Carnosine+OGD/R group: $9.6 \% \pm 3.24 \%, P<0.05)$, indicating that carnosine induces $\mathrm{G}_{1}$ arrest in reactive astrocytes induced by OGD/R by inhibiting the $G_{1}$-to-S phase transition. Next, we measured the protein expression levels of cyclin D1, the critical transition protein that is required for progression from $G_{1}$ into $S$ phase ${ }^{[23]}$. The results showed that carnosine treatment reversed the increase in cyclin D1 protein expression induced by OGD/ $\mathrm{R}$ (Figure 3C, 3D). Thus, these results suggest that carnosine inhibited reactive gliosis of astrocytes may be due at least in part to its ability to suppress the $\mathrm{G}_{1}$-to-S phase transition.

Effect of carnosine on mitochondrial bioenergetics and glycolysis of cultured astrocytes exposed to OGD/R

Energy metabolism is also closely related to cell proliferation.
Therefore, in this study, we also investigated the effects of carnosine on the oxygen consumption rate and extracellular acidification rate in cultured astrocytes exposed to OGD/R. The results showed that OGD for $2 \mathrm{~h}$ and recovery for $24 \mathrm{~h}$ increased the basal cellular oxygen consumption rates (OCRs) and extracellular acidification rates (ECARs) to $341.4 \pm 56.3$ pmol $\cdot \mathrm{min}^{-1} \cdot \mu \mathrm{g}^{-1}$ protein $(138 \%$ of control, Figure $4 \mathrm{~A}, 4 \mathrm{C})$, and $91.58 \pm 18.21 \mathrm{mpH} \cdot \mathrm{min}^{-1} \cdot \mu \mathrm{g}^{-1}$ protein $(\sim 217 \%$ of control, Figure 4B, 4D), respectively. The mitochondrial respiration and non-mitochondrial respiration of cultured astrocytes exposed to OGD/R were both increased significantly. In addition, OGD/R treatment caused a marked increase both in ATP-linked respiration and proton leak of cultured astrocytes (Figure 4C). Carnosine treatment did not affect the multiple parameters of mitochondrial respiration (Figure 4C), whereas it markedly reversed the increase in ECARs induced by OGD/ $\mathrm{R}$ in cultured astrocytes (Figure 4D). In addition, we found that carnosine treatment significantly reversed the increase in ATP production induced by OGD/R in cultured astrocytes (Figure 4E).

\section{Carnosine suppressed migration of astrocytes exposed OGD/R in vitro}

A cell migration assay was used to ascertain the effect of carnosine on the migration of cultured astrocytes. Compared with the control group, the number of astrocytes migrating through the film increased significantly (274\% of control) after OGD/R. However, when $5 \mathrm{mmol} / \mathrm{L}$ carnosine was added to the culture medium, the increased migration could partly be inhibited (53\% of OGD/R group, Figure 5A, 5B). These results suggested that carnosine is probably involved in regulating migration of astrocytes exposed to OGD/R. Then, we used Western blotting technology to test the expression level of matrix metalloproteinases-9 (MMP-9), which is closely related to cell migration ${ }^{[24]}$. The results showed that the expression level of MMP-9 in astrocytes after OGD/R increased significantly when compared with control group, while carnosine treatment could cut high levels of MMP-9 in cells after OGD/ $\mathrm{R}$ (Figure 5C,5D). Thus, the effect of carnosine on migration levels of reactive astrocytes may be related to its modulation effect on MMP-9.

\section{Carnosine improved neurite growth in neuron-astrocyte co- cultures}

To study the effect of carnosine on neurite growth, we also detected the neurite length by immunofluorescence staining of class III $\beta$-tubulin in control, OGD/R and carnosine-treated cells. We found that the mean length of neurites was prominently shorter than the controls following 2-h OGD and 24-h recovery, whereas carnosine treatment significantly improved the inhibited growth of neurites (Control group: 223.87 \pm 59.06 , OGD/R group: 78.92 \pm 43.41 , OGD/R+Carnosine group: 163.75 $\pm 59.47, P<0.01$, Figure 6).

\section{Discussion}

Glial scar formation in the later stage of cerebral ischemia is 

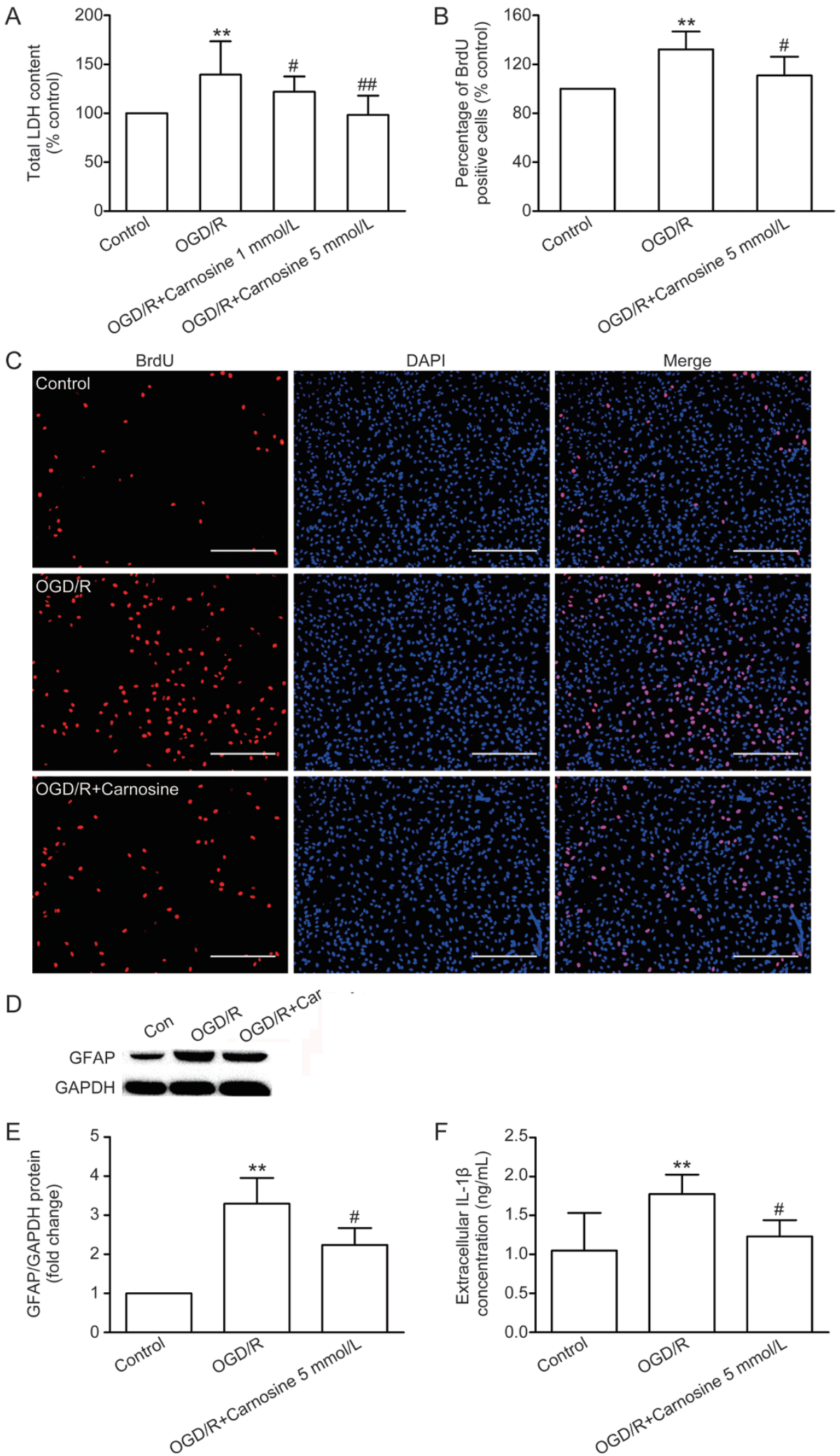

Figure 2. Carnosine suppressed reactive gliosis of astrocytes induced by OGD/R. Astrocytes were pre-treated with carnosine (1 or $5 \mathrm{mmol} / \mathrm{L})$ for $30 \mathrm{~min}$ and were then exposed to OGD for $2 \mathrm{~h}$ and recovery for $24 \mathrm{~h}$. At the end of OGD/R, the total LDH content was assayed (A), and double immunofluorescence of BrdU (red) and DAPI (blue) in astrocytes was performed. Proportions of proliferation cells in each group after OGD/R were statistically analyzed (B, C). GFAP protein levels were also analyzed by Western blot after OGD/R (D). Densitometric analysis of bands for relative GFAP protein expression levels is shown (E). Extracellular IL-1 $1 \beta$ level was measured by ELISA (F). Data represent the mean $\pm S D$. ${ }^{* *} P<0.01$ vs control group. ${ }^{\#} P<0.05,{ }^{\#} P<0.01$ vs OGD/R group. Scale bar, $200 \mu \mathrm{m}$. 
A

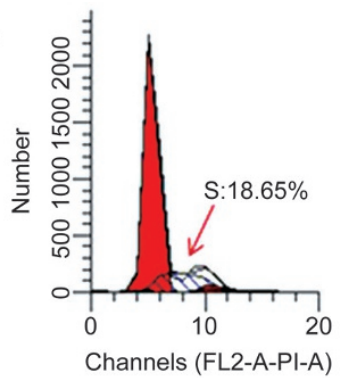

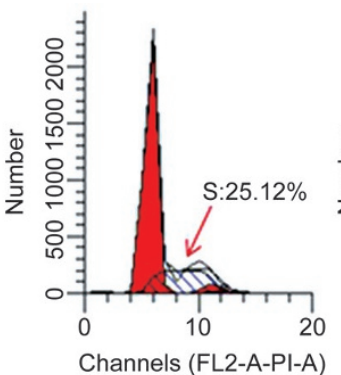

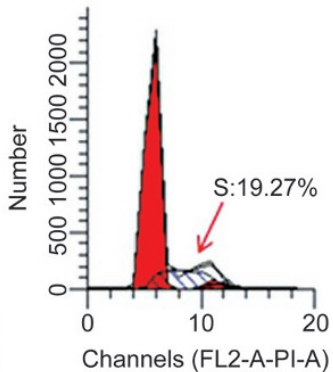

B

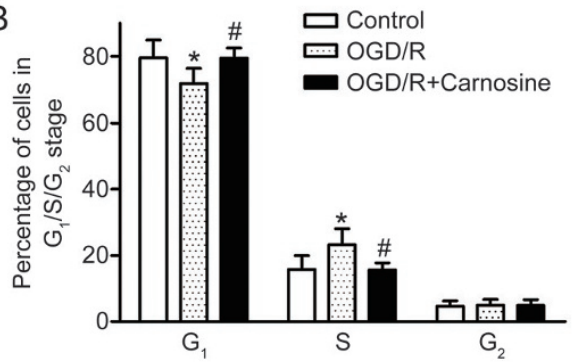

C

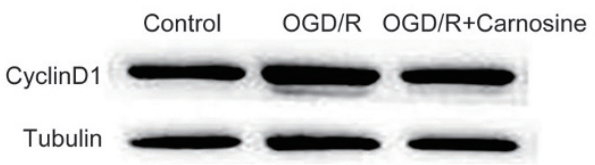

Figure 3. Carnosine inhibited cell cycle progression in cultured astrocytes exposed to OGD/R. Representative pictures of flow cytometric analysis of control, OGD/R, and carnosine-treated cells (A). Statistical analysis of the percentage of cells in each cell cycle phase (B). Western blot analysis of the expression level of cyclin D1 in astrocytes exposed to OGD/R and carnosine treatment (C). Densitometric analysis of bands for relative cyclin D1 protein expression level (D). Data represent the mean \pm SD. ${ }^{*} P<0.05$ vs control group. ${ }^{\#} P<0.05$ vs OGD/R group.

an important factor affecting the nervous function recovery of patients. In the current study, we demonstrated for the first time that carnosine can inhibit the proliferation and migration of reactive astrocytes induced by $\mathrm{OGD} / \mathrm{R}$ in vitro. Moreover, we also found a direct effect of carnosine on axonal regeneration in neuron/astrocyte co-cultures exposed to OGD/R.

Cerebral ischemia-induced widespread progressive alterations of astrocytes, which include cell hypertrophy, up-regulation of GFAP expression, and increased proliferation capability, are commonly known as reactive astrogliosis ${ }^{[25]}$. Here, we found that after $2 \mathrm{~h}$ OGD and $24 \mathrm{~h}$ reperfusion, the percentage of $\mathrm{BrdU}^{+}$astrocytes significantly increased, and the expression of GFAP and the release of IL- $1 \beta$ were also markedly up-regulated, indicating that $\mathrm{OGD} / \mathrm{R}$ can induce astrocytes to present glial scar characteristics. Therefore, an OGD/R-induced in vitro model of glial scar that can be controlled easily and conveniently can be used to explore the mechanisms underlying glial scar formation and to find new ways to improve brain repair and functional recovery after stroke.

Recently, an inhibitory effect of carnosine on the proliferation of several transformed and neoplastic cells has been reported by our group and others ${ }^{[13,26-28]}$. In the present study, we found that carnosine treatment significantly reduced the number of $\mathrm{BrdU}^{+}$astrocytes $24 \mathrm{~h}$ after OGD. Simultaneously, the enhanced expression of GFAP protein induced by OGD/R was also partly reversed by carnosine, indicating that car- nosine treatment also inhibited the proliferation of reactive astrocytes induced by OGD/R. It has been reported that carnosine acts as a regulator of cell cycle in cancer cells. Iovine $e t$ al showed that carnosine induces $\mathrm{G}_{1}$ arrest in human HCT116 colon cancer cells by inhibiting the $\mathrm{G}_{1} / \mathrm{S}$ phase transition ${ }^{[28]}$. Interestingly, we also found that carnosine was capable of suppressing the transformation of reactive astrocytes from $\mathrm{G}_{1}$ phase to $\mathrm{S}$ phase and decreasing the cyclin D1 protein level. Thus, besides cancer cells, carnosine may also inhibit the proliferation of normal cells with increased proliferative capacity, and the underlying mechanism may be at least partly due to its action on the cell cycle and cyclin-dependent kinases.

It was also reported that the anti-proliferation effect of carnosine on tumor and cancer cells is related to its ability to suppress glycolytic activity and/or mitochondrial energy of the cells ${ }^{[13,26]}$. Our recent study also revealed that carnosine exerts biphasic modulation on energy metabolism of cultured cortical astrocytes under normal and ischemic conditions ${ }^{[18]}$. Thus, it seems that cellular energy metabolism is considered a target of carnosine. Data from the Seahorse XF96 Extracellular Flux Analyzer demonstrated that OGD/R-induced reactive astrocytes had higher ATP-linked oxygen consumption rates and basal ECARs, indicating that both mitochondrial bioenergetics and glycolysis pathways are activated in reactive astrocytes for the increased energy demand to facilitate cell proliferation. Carnosine could not reverse the increase in mitochondrial 

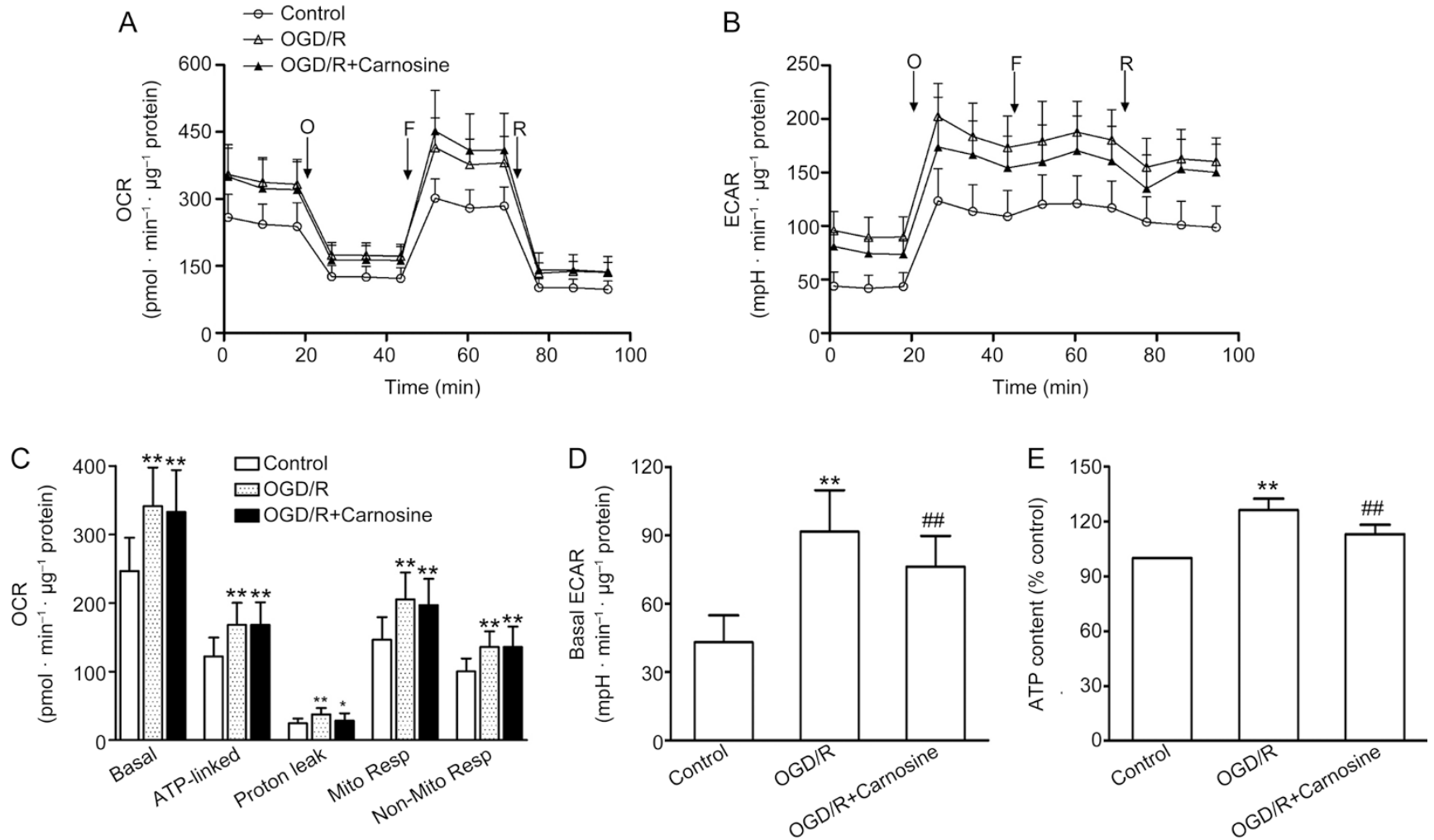

Figure 4. Effect of carnosine on astrocytic energy metabolism in cultured astrocytes exposed to OGD/R. Real-time analysis of OCRs (A) and ECARs (B) of cultured astrocytes exposed to OGD/R and carnosine treatment by perturbing them with small molecule metabolic modulators. Oligomycin ( $\mathrm{O}$, $1 \mu \mathrm{g} / \mathrm{mL}), \operatorname{FCCP}(F, 1 \mu \mathrm{mol} / \mathrm{L})$, and rotenone (R, $1 \mu \mathrm{mol} / \mathrm{L})$ were injected sequentially at the indicated time points into each well containing astrocytes after baseline rate measurement. Multiple mitochondrial function parameters, including basal OCRs, ATP-linked OCRs, proton leak, mitochondrial respiration and non-mitochondrial respiration OCRs, are shown (C). OCRs and ECARs were normalized to total protein/well after completion of the assay. Carnosine treatment decreased the cellular ATP level in astrocytes exposed to OGD/R (E). Intracellular ATP level was measured at the end of OGD/R. Data represent the mean \pm SD. ${ }^{*} P<0.05,{ }^{* *} P<0.01$ vs control group. ${ }^{\# \#} P<0.01$ vs OGD/R group.

respiration, but it significantly reversed the increase in ECARs in reactive astrocytes. Carnosine has been recognized as a $\mathrm{pH}$ buffer, and carnosine in the culture medium can affect monitoring for ECARs. Therefore, in this study, we washed the cells carefully to avoid residual carnosine before the cells were switched to unbuffered DMEM. In the current study, the data also showed that carnosine can partly reverse the increase in ATP production induced by OGD/R in reactive astrocytes. Thus, the data indicate that it is the glycolysis, but not the mitochondrial bioenergetics pathway, involved in the carnosine action on the proliferation of reactive astrocytes. Stephanie et al have reported that carnosine adversely affects fermentative growth of Saccharomyces cerevisiae in glucose, while aerobic growth on glycerol is not inhibited, suggesting that the metabolism-dependent effects of carnosine are mediated by different mechanisms ${ }^{[29]}$. In addition, it has been reported that during mid-to-late $G_{1}$, a nutrient-sensitive cell growth checkpoint controls progression to $S$ phase. The cell must prepare for division in $\mathrm{G}_{1}$ by synthesizing macromolecules needed for biomass duplication. Mitotically committed cells thus upregulate the glycolytic activator fructose-2,6-bisphosphatase 3 (PFKFB3) at the nutrient-sensitive checkpoint and block glycolysis to impair the passage through this restriction point.
Thus, the glycolysis pathway may be involved in carnosine's action on $\mathrm{G}_{1}$ arrest/decreased expression of cyclin D1 in reactive astrocytes.

Astrocyte migration is a key step in glial scar formation after cerebral ischemia ${ }^{[30]}$, and weaker astrocyte migratory activity contributes to neuroprotection ${ }^{[31]}$. In the current study, we found that the migratory activity of reactive astrocytes could be suppressed by carnosine. It has been reported that astrocyte migration is regulated by multiple factors, such as MMP-9, which degrades extracellular matrix components and plays important roles in a variety of biological and pathological processes ${ }^{[32]}$. Here, we found that OGD/R markedly upregulated MMP-9 expression in astrocytes, whereas expression of MMP-9 was significantly suppressed by carnosine treatment. Our results are consistent with previous findings that carnosine inhibits metastasis of SK-Hep-1 cells via inhibition of MMP-9 expression ${ }^{[33]}$. Thus, our data suggest that in addition to cancer cells, carnosine may also inhibit the migration of normal cells by reducing MMP-9 expression in these cells.

Previously, it was demonstrated that the growth of neuronal axons is often arrested after stroke $\mathrm{e}^{[3,35]}$. In the current study, following 24-h recovery after OGD, the neurons exposed to OGD with astrocytes showed abortive regeneration, as they 

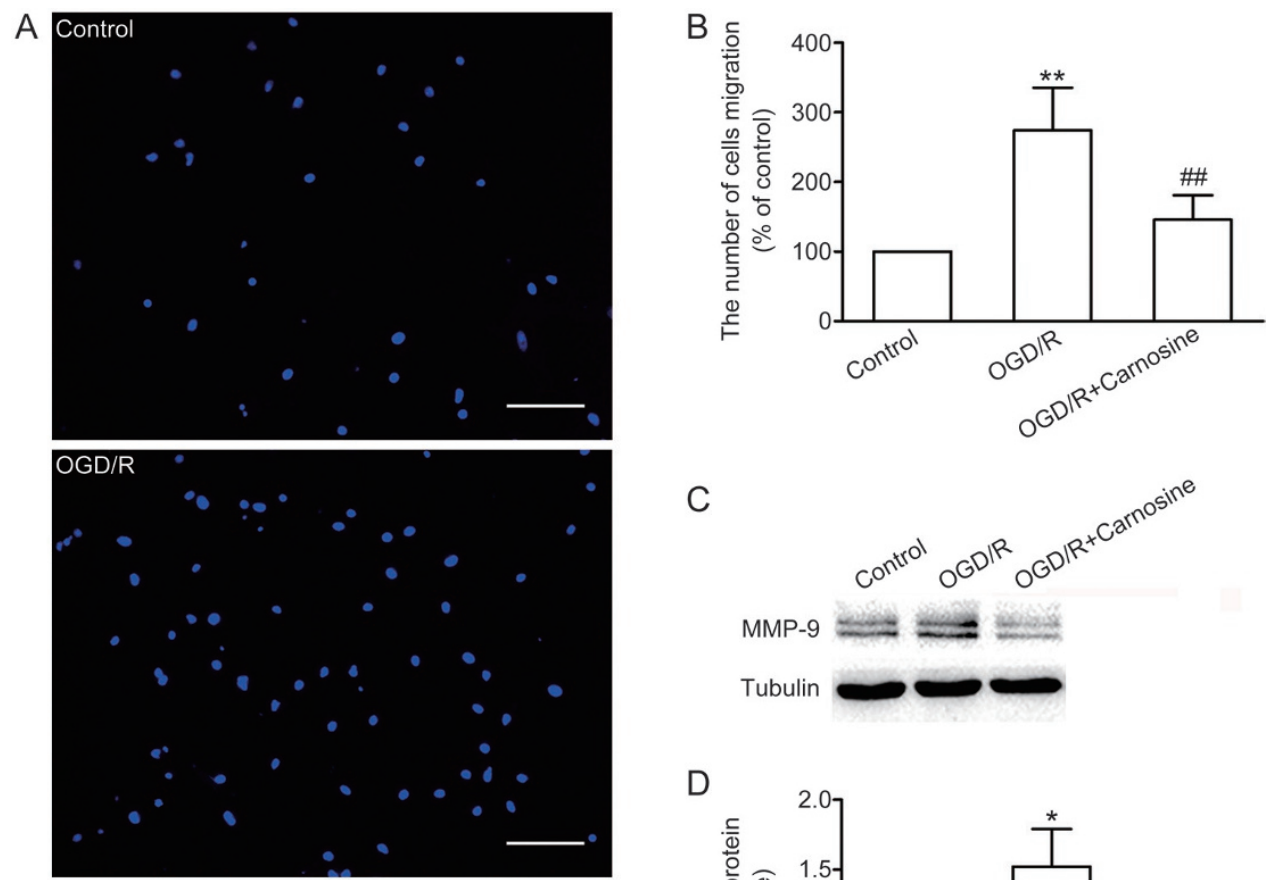

C
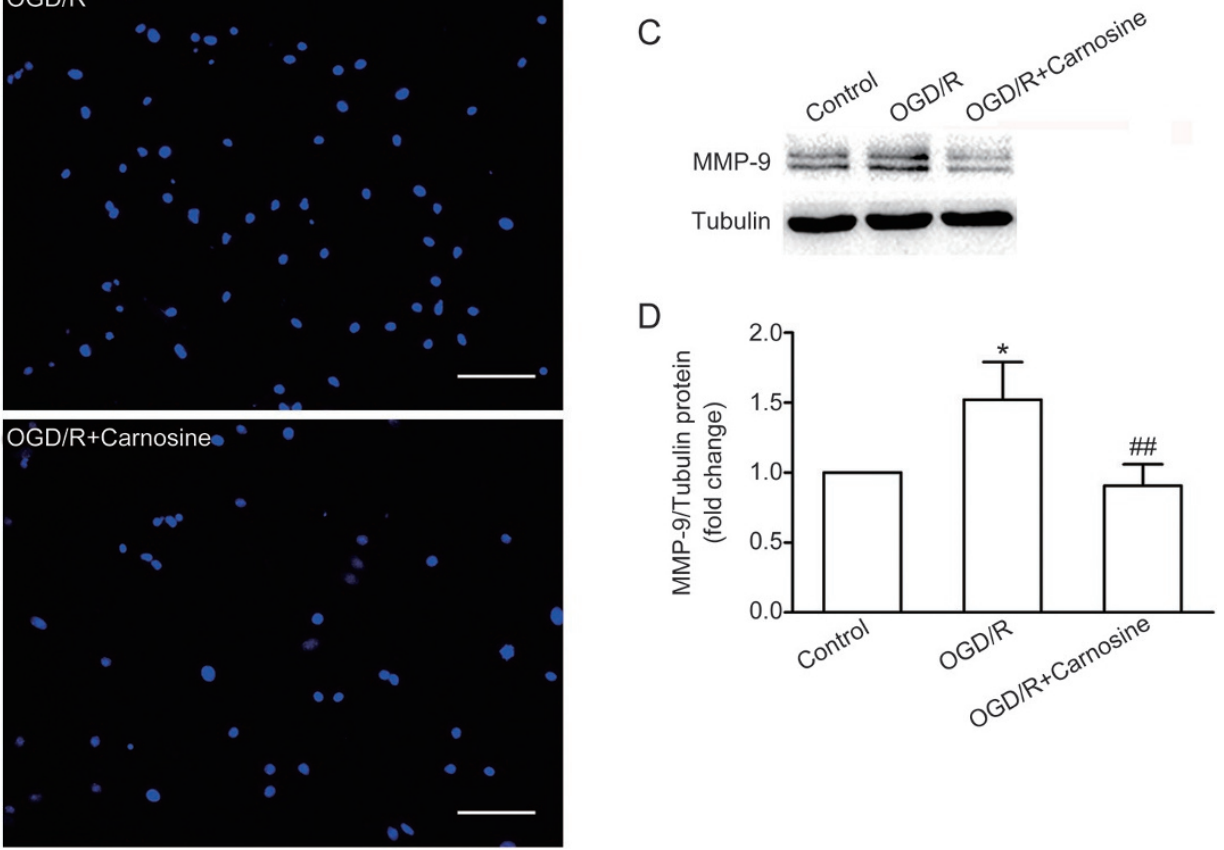

Figure 5. Carnosine inhibited astrocyte migration after OGD/R. Photomicrographs of DAPI-stained astrocytes that crossed the filters with 8- $\mu$ m pore size (A). Statistical analysis of relative migration rates of astrocytes in the control, OGD/R and carnosine-treated groups (B). Western blot analysis of the expression level of MMP-9 protein in astrocytes exposed to OGD/R and carnosine treatment (C). Densitometric analysis of bands for relative MMP-9 protein expression level (D). Data represent the mean \pm SD. ${ }^{*} P<0.05,{ }^{* *} P<0.01$ vs control group. ${ }^{\# \#} P<0.01$ vs 0 GD/R group.

attained much shorter mean neurite lengths, and their morphologies were also consistent with neurons responding to an inhibitory environment induced by other injuries ${ }^{[6,34]}$. Thus, these data suggest that astrocytes at 24-h recovery after 2-h OGD exhibit a glial scar-like action to inhibit neuron growth. However, Wang et al reported that astrocytes at 48-h recovery after OGD present a glial scar-like action ${ }^{[36]}$. The discrepant results from these two different experiments are probably due to the use of distinctly different neuron/astrocyte co-culture models. In the current study, we also found that the neurite growth inhibition was markedly reversed by carnosine. Boato et al reported that increased local levels of IL- $1 \beta$ exerted detrimental effects on lesion size and glial activation as well as on the plasticity of CNS axons in vivo after injury ${ }^{[22]}$. Thus, the positive action of carnosine on neurite growth was probably partly due to the inhibitory effect of carnosine on the release of IL-1 $\beta$ from the activated astrocytes, or other direct or indirect actions of carnosine on the proliferation and migration of the reactive astrocytes.

In conclusion, our results showed that carnosine reduced proliferation and migration of reactive astrocytes induced by OGD/R by arresting the cell cycle at the $\mathrm{G}_{1} / \mathrm{S}$ phase, suppressing glycolysis and ATP production and regulating MMP-9 expression. Also, carnosine treatment led to better axonal regrowth in neuron/astrocyte co-cultures under ischemic conditions. Taken together, these results suggest that carnosine may be a good candidate as a therapeutic agent for brain functional recovery following cerebral ischemia.

\section{Acknowledgements}

This work was supported by the National Natural Science Foundation of China (81571289).

\section{Author contribution}

Yao SHEN and Jian-xin LYU conceived and designed the experiments; Li OU-YANG and Yao SHEN wrote the manu- 
A
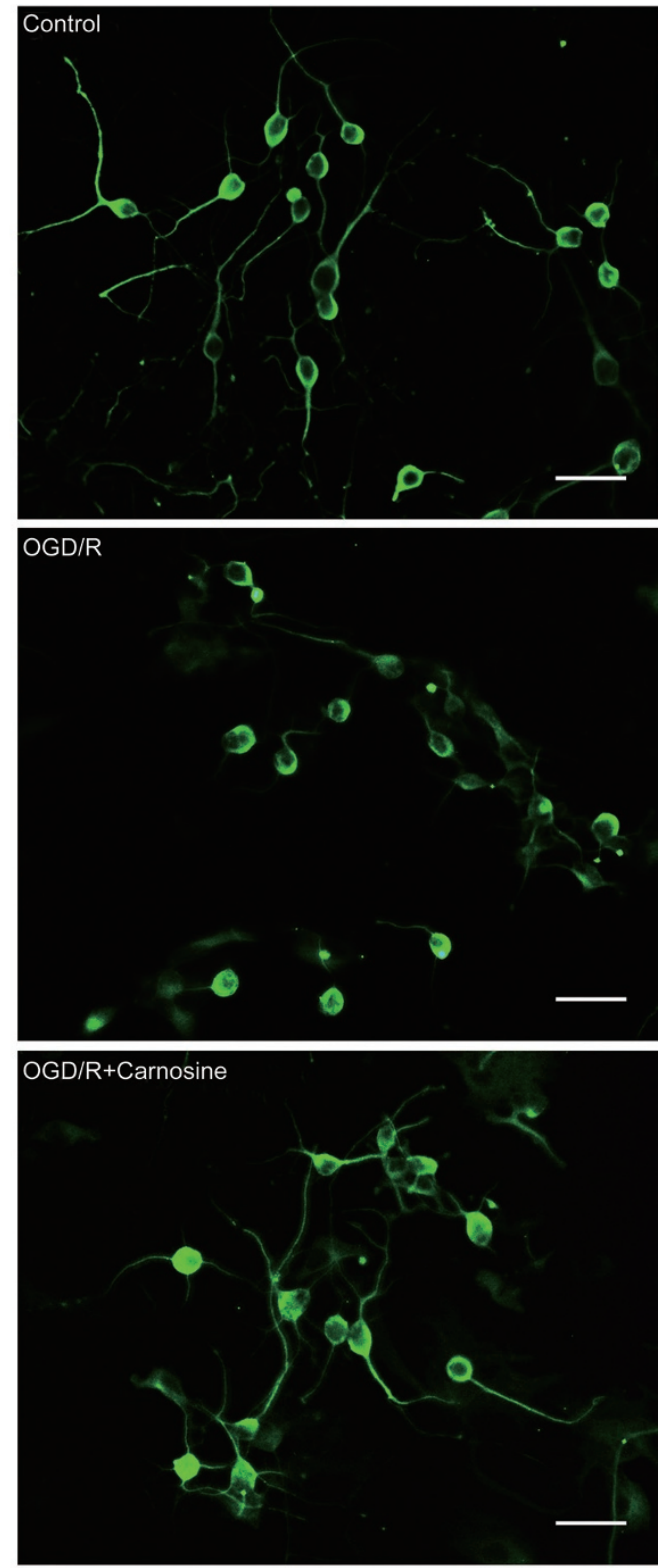

B

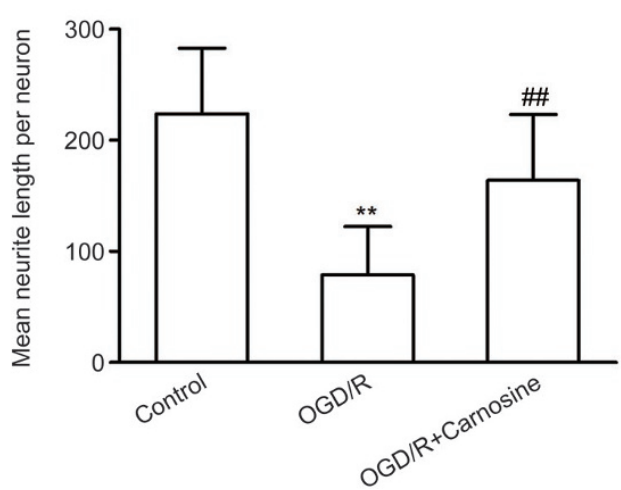

Figure 6. Carnosine increased neurite length in astrocyte/neuron cocultures exposed to OGD/R. Beta III tubulin immunostaining of cocultured neurons (A). Scale bar, $200 \mu \mathrm{m}$. Quantification of mean neurite length per neuron (B). Data represent the mean \pm SD. ${ }^{* *} P<0.01$ vs control group. ${ }^{\#} P<0.01$ vs OGD/R group. script; Li OU-YANG, Yuan LIU and Bing-yu WANG performed experiments and analyzed the data; Pei CAO, Jing-jing ZHANG and Yu-yan HUANG performed the experiments.

\section{References}

1 Huang L, Wu ZB, Zhuge Q, Zheng W, Shao B, Wang B, et al. Glial scar formation occurs in the human brain after ischemic stroke. Int J Med Sci 2014; 11: 344-8.

2 Cregg JM, DePaul MA, Filous AR, Lang BT, Tran A, Silver J. Functional regeneration beyond the glial scar. Exp Neurol 2014; 253: 197-207.

3 Yiu G, He Z. Glial inhibition of CNS axon regeneration. Nat Rev Neurosci 2006; 7: 617-27.

4 Raposo C, Schwartz M. Glial scar and immune cell involvement in tissue remodeling and repair following acute CNS injuries. Glia 2014; 62: 1895-904.

5 Tan AM, Zhang W, Levine JM. NG2: a component of the glial scar that inhibits axon growth. J Anat 2005; 207: 717-25.

6 Silver J, Miller JH. Regeneration beyond the glial scar. Nat Rev Neurosci 2004; 5: 146-56.

7 Rolls A, Shechter R, Schwartz M. The bright side of the glial scar in CNS repair. Nat Rev Neurosci 2009; 10: 235-41.

8 Choudhury GR, Ding S. Reactive astrocytes and therapeutic potential in focal ischemic stroke. Neurobiol Dis 2016; 85: 234-44.

9 Boldyrev AA. Carnosine: new concept for the function of an old molecule. Biochemistry (Mosc) 2012; 77: 313-26.

10 Kamal MA, Jiang $\mathrm{H}, \mathrm{Hu} \mathrm{Y}$, Keep RF, Smith DE. Influence of genetic knockout of Pept2 on the in vivo disposition of endogenous and exogenous carnosine in wild-type and Pept2 null mice. Am J Physiol Regul Integr Comp Physiol 2009; 296: R986-91.

11 Rajanikant GK, Zemke D, Senut MC, Frenkel MB, Chen AF, Gupta R, et al. Carnosine is neuroprotective against permanent focal cerebral ischemia in mice. Stroke 2007; 38: 3023-31.

12 Gleichman AJ, Carmichael ST. Astrocytic therapies for neuronal repair in stroke. Neurosci Lett 2014; 565: 47-52.

13 Shen Y, Yang J, Li J, Shi X, Ouyang L, Tian Y, et al. Carnosine inhibits the proliferation of human gastric cancer SGC-7901 cells through both of the mitochondrial respiration and glycolysis pathways. PLoS One 2014; 9: e104632.

14 Pekcetin C, Kiray M, Ergur BU, Tugyan K, Bagriyanik HA, Erbil G, et al. Carnosine attenuates oxidative stress and apoptosis in transient cerebral ischemia in rats. Acta Biol Hung 2009; 60: 137-48.

15 Shen Y, Zhang S, Fu L, Hu W, Chen Z. Carnosine attenuates mast cell degranulation and histamine release induced by oxygen-glucose deprivation. Cell Biochem Funct 2008; 26: 334-8.

16 Hipkiss AR, Cartwright SP, Bromley C, Gross SR, Bill RM. Carnosine: can understanding its actions on energy metabolism and protein homeostasis inform its therapeutic potential? Chem Cent J 2013; 7 : 38.

17 Hipkiss AR. Energy metabolism, proteotoxic stress and age-related dysfunction - protection by carnosine. Mol Aspects Med 2011; 32: 267-78.

18 Shen Y, Tian Y, Yang J, Shi X, Ouyang L, Gao J, et al. Dual effects of carnosine on energy metabolism of cultured cortical astrocytes under normal and ischemic conditions. Regul Pept 2014; 192-193: 45-52.

19 Shen Y, Tian Y, Shi X, Yang J, Ouyang L, Gao J, et al. Exposure to high glutamate concentration activates aerobic glycolysis but inhibits ATPlinked respiration in cultured cortical astrocytes. Cell Biochem Funct 2014; 32: 530-7.

20 Beazley KE, Zhang T, Lima F, Pozharskaya T, Niger C, Tzitzikov E, et al. Implication for transglutaminase 2-mediated activation of beta- 
catenin signaling in neointimal vascular smooth muscle cells in chronic cardiac allograft rejection. J Heart Lung Transplant 2012; 31: 1009-17.

21 Shen Y, He P, Fan YY, Zhang JX, Yan HJ, Hu WW, et al. Carnosine protects against permanent cerebral ischemia in histidine decarboxylase knockout mice by reducing glutamate excitotoxicity. Free Radic Biol Med 2010; 48: 727-35.

22 Boato F, Rosenberger K, Nelissen S, Geboes L, Peters EM, Nitsch R, et al. Absence of IL-1beta positively affects neurological outcome, lesion development and axonal plasticity after spinal cord injury. J Neuroinflammation 2013; 10: 6.

23 Di Giovanni S, Movsesyan V, Ahmed F, Cernak I, Schinelli S, Stoica B, et al. Cell cycle inhibition provides neuroprotection and reduces glial proliferation and scar formation after traumatic brain injury. Proc Natl Acad Sci U S A 2005; 102: 8333-8.

24 Copin JC, Gasche Y. Matrix metalloproteinase-9 deficiency has no effect on glial scar formation after transient focal cerebral ischemia in mouse. Brain Res 2007; 1150: 167-73.

25 Sofroniew MV. Molecular dissection of reactive astrogliosis and glial scar formation. Trends Neurosci 2009; 32: 638-47.

26 Hipkiss AR, Gaunitz F. Inhibition of tumour cell growth by carnosine: some possible mechanisms. Amino Acids 2014; 46: 327-37.

27 Holliday R, McFarland GA. Inhibition of the growth of transformed and neoplastic cells by the dipeptide carnosine. Br J Cancer 1996; 73: 966-71.

28 Iovine B, Iannella ML, Nocella F, Pricolo MR, Bevilacqua MA. Carnosine inhibits KRAS-mediated HCT116 proliferation by affecting
ATP and ROS production. Cancer Lett 2012; 315: 122-8.

29 Cartwright SP, Bill RM, Hipkiss AR. L-carnosine affects the growth of Saccharomyces cerevisiae in a metabolism-dependent manner. PLoS One 2012; 7: e45006.

30 Li CY, Li X, Liu SF, Qu WS, Wang W, Tian DS. Inhibition of mTOR pathway restrains astrocyte proliferation, migration and production of inflammatory mediators after oxygen-glucose deprivation and reoxygenation. Neurochem Int 2015; 83-84: 9-18.

31 Liu T, Xue CC, Shi YL, Bai XJ, Li ZF, Yi CL. Overexpression of mitofusin 2 inhibits reactive astrogliosis proliferation in vitro. Neurosci Lett 2014; 579: 24-9.

32 Hsieh HL, Yang SH, Lee TH, Fang JY, Lin CF. Evaluation of antiinflammatory effects of helminthostachys zeylanica extracts via inhibiting bradykinin-induced MMP-9 expression in brain astrocytes. Mol Neurobiol 2016; 53: 5995-6005.

33 Chuang $\mathrm{CH}$, Hu ML. L-carnosine inhibits metastasis of SK-Hep-1 cells by inhibition of matrix metaoproteinase- 9 expression and induction of an antimetastatic gene, nm23-H1. Nutr Cancer 2008; 60: 526-33.

34 Wanner IB, Deik A, Torres M, Rosendahl A, Neary JT, Lemmon VP, et al. A new in vitro model of the glial scar inhibits axon growth. Glia 2008; 56: 1691-709.

35 Lee JK, Kim JE, Sivula M, Strittmatter SM. Nogo receptor antagonism promotes stroke recovery by enhancing axonal plasticity. J Neurosci 2004; 24: 6209-17.

36 Wang R, Zhang X, Zhang J, Fan Y, Shen Y, Hu W, et al. Oxygen-glucose deprivation induced glial scar-like change in astrocytes. PLoS One 2012; 7: e37574. 